\title{
Impact of Proteinase 3 versus Myeloperoxidase Positivity on Risk of End-Stage Renal Disease in ANCA-Associated Glomerulonephritis Stratified by Histological Classification: A Population-Based Cohort Study
}

\author{
Vilde Solbakken $\left(\mathbb{D},{ }^{1}\right.$ Anne-Siri Fismen, ${ }^{2}$ Leif Bostad, ${ }^{3}$ and Rune Bjørneklett $\mathbb{D}^{1,4}$ \\ ${ }^{1}$ Department of Clinical Medicine, University of Bergen, Bergen, Norway \\ ${ }^{2}$ Faculty of Health and Social Sciences, Western Norway University of Applied Sciences, Bergen, Norway \\ ${ }^{3}$ Department of Pathology, Haukeland University Hospital, Bergen, Norway \\ ${ }^{4}$ Emergency Care Clinic, Haukeland University Hospital, Bergen, Norway
}

Correspondence should be addressed to Rune Bjørneklett; rune.bjoerneklett@helse-bergen.no

Received 14 January 2018; Accepted 16 April 2018; Published 9 May 2018

Academic Editor: Eric A. Singer

Copyright ( 2018 Vilde Solbakken et al. This is an open access article distributed under the Creative Commons Attribution License, which permits unrestricted use, distribution, and reproduction in any medium, provided the original work is properly cited.

\begin{abstract}
Background. End-stage renal disease (ESRD) risk in patients with antineutrophil cytoplasmic antibody- (ANCA-) associated glomerulonephritis (ANCA-GN) according to ANCA serotype and stratified by histological classification has not been previously investigated. Methods. Patients from the Norwegian Kidney Biopsy Registry (NKBR) between 1991 and 2012 who had biopsy-verified pauci-immune glomerulonephritis and positive antineutrophil cytoplasmic antibody serology were included. Cases with ESRD during follow-up were identified in the Norwegian Renal Registry. ESRD-free survival with proteinase 3 (PR3) versus myeloperoxidase- (MPO-) ANCA positivity stratified into 4 histological classes was investigated. Results. Three hundred fifty-eight patients, of whom 87 progressed to ESRD during follow-up, were included. Patients with PR3- as compared to MPOANCA were younger (58 versus 64 years, $p=0.001$ ), had a higher percentage of males (62 versus $41 \%, p<0.001$ ), had a lower percentage with a sclerozing glomerulonephritis pattern (4 versus $16 \%, p<0.001$ ), and had a significantly higher cumulative ESRD-free survival ( 90 versus $80 \%, p=0.007$ ) at 1 -year follow-up. No significant differences in cumulative ESRD-free survival with PR3- as compared to MPO-ANCA were observed by histological stratification. Conclusion. Advanced glomerular sclerosis is found more frequently in patients with MPO-ANCA, explaining the higher risk of ESRD. ANCA serotypes have no impact on prognosis of patients with similar histological findings.
\end{abstract}

\section{Introduction}

Antineutrophil cytoplasmic antibody- (ANCA-) associated vasculitis (AAV) typically affecting small vessels and kidney involvement in the form of pauci-immune glomerulonephritis (ANCA-GN) is common [1]. Even with modern treatment, AAV is associated with significant morbidity and mortality; moreover, in patients with ANCA-GN, progression to end-stage renal disease (ESRD) resulting in the need for chronic dialysis treatment or kidney transplantation is a particular concern [2]. Although ANCA-GN from a clinical point of view usually is regarded as one disease, it is associated with 2 different autoantibodies, proteinase 3 (PR3) and myeloperoxidase (MPO). A number of differences between patients with PR3- as compared to MPO-AAV have been described [3]. Differing genetic associations, $[4,5]$ as well as substantial sex differences, have been found $[6,7]$. In some studies, worse baseline renal function in patients with MPO- as compared to PR3-ANCAGN has been reported [8, 9]. The histopathological findings in ANCA-GN are qualitatively similar, irrespective of ANCA type [10,11]. However, a few studies have noticed quantitative differences in the form of a higher percentage of normal glomeruli in PR3-ANCA-GN and 
more fibrotic changes in MPO-ANCA-GN [10, 12-14]. Despite a higher risk of relapse [15-17], renal prognosis has often been found better in PR3- than in MPOAAGN [18-20]. However, it is unclear whether the poorer prognoses associated with MPO- as compared to PR3ANCA-GN are caused by more advanced disease at baseline $[7,8,21-24]$ or due to differences in the nature of inflammation associated with these 2 different autoantibody serotypes [25-27].

The histological classification of patients with ANCAGN into 4 groups, focal ( $\geq 50 \%$ normal glomeruli), crescentic ( $\geq 50 \%$ cellular crescents), mixed $(<50 \%$ normal, $<50 \%$ crescentic, and $<50 \%$ globally sclerotic glomeruli), and sclerotic ( $\geq 50 \%$ globally sclerotic glomeruli), is useful for stratifying patients according to the risk of progression to ESRD [7, 20, 28-31]. To our knowledge, data comparing renal disease and patient survival with PR3- as compared to MPO-ANCA-GN, in cases with similar histological classification, have not been previously published. Here, using data from the Norwegian Kidney Biopsy Registry, we have compared baseline characteristics, histological findings, and outcomes stratified for histological classification in patients with ANCA-GN divided by PR3- as compared to MPO-ANCA positivity.

\section{Material and Methods}

This study was approved by the Regional Committee for Medical and Health Research Ethics. Data sources used are based on informed consent.

In the present investigation, we included patients with ANCA-GN from a previous study cohort used by our group [29]. Identification of the study cohort, baseline data, scoring of patients according to the histologic classification model of ANCA-GN, definition of observation period, and endpoints were described in detail earlier and only briefly reviewed here.

All patients with a biopsy containing at least 3 glomeruli and histologically verified ANCA-GN in the Norwegian Kidney Biopsy Registry (NKBR) from 1991 to 2012 were included in the study cohort. The criteria for ANCA-GN were the presence of pauci-immune necrotizing glomerulonephritis and a positive ANCA titer. Baseline data, including sex, age, ANCA serotype (determined by indirect immunofluorescence and/or enzyme linked immunosorbent assay methods), estimated glomerular filtration rate (eGFR), serum albumin, systolic and diastolic blood pressure, and proteinuria were obtained from the NKBR. An experienced renal pathologist (LB) classified all cases according to the ANCAGN histological classification scheme. The observation period was from the time of biopsy to the first event, ESRD, death, or end of 2012. Furthermore, the observation period was stratified according to the induction $(\leq 1$ year after biopsy) and the remission ( $>1$ year after biopsy) phases. The primary endpoint of this study was ESRD, defined as the initiation of chronic renal replacement therapy in the form of dialysis or renal transplantation. Cases with ESRD were identified by the linkage of the study cohort with the Norwegian Renal Registry. The secondary endpoint was death. These were identified by linking the study cohort with the Population Registry of Norway and also used to define a combined endpoint, which consists of patients with ESRD or those who died during follow-up, whichever came first.

First, we analyzed baseline data stratified according to the MPO-/PR3-ANCA serotype. Second, we analyzed 1- and 5year cumulative ESRD-free survival stratified for MPO and PR3 positivity and specifically for each histological class of ANCA-GN using Kaplan-Meier statistics. Finally, Cox regression statistics was used to calculate the hazard ratio (HR) of ESRD with MPO- versus PR3-ANCA after adjustment for histological class, sex, and age. In these analyses, deaths without ESRD were treated as censored events. The analyses were then repeated using the secondary endpoints: ESRD/death (deaths treated as an event) and deaths (deaths with ESRD included as events). For significance testing, Chi-square test was used with categorical variables, MannWhitney $U$ test for continuous variables, and Log-rank test for comparisons of survival. SPSS, version 24 was used for statistical analyses.

\section{Results}

Three hundred fifty-eight patients were identified and included in the study cohort. One hundred ninety-three (54\%) were PR3-ANCA positive. One hundred eighty-seven $(52 \%)$ were male, and their mean age was 60 years $(\mathrm{SD}=17)$, mean eGFR was $34 \mathrm{ml} / \mathrm{min} / 1.73 \mathrm{~m}^{2}(\mathrm{SD}=30)$, and mean percentage glomeruli without crescents or global sclerosis was $36 \%(\mathrm{SD}=29)$. Other baseline characteristics are shown in Table 1. Median observation period was 3.5 years $(25$ th -75 th percentiles $=0.6-7.7$ years $)$ and the total number of patient years was 1716. During the short followup period ( $\leq 1$ year), $50(14 \%)$ of the patients underwent chronic renal replacement therapy and 47 (13\%) patients without ESRD died. Two hundred sixty-one $(73 \%)$ patients survived $>1$ year after the diagnosis of ANCA-GN without ESRD, 37 (10\%) later progressed to ESRD, 31 (9\%) without ESRD died, and 193 (54\%) were alive without ESRD by the end of 2012. Forty-eight of 87 patients with ESRD were alive by the end of 2012 .

Baseline data stratified according to ANCA serotype are shown in Table 1. A significant sex difference was observed. PR3-ANCA was found in $62 \%$ of male and $41 \%$ of female patients $(p<0.001)$. Mean age in patients with PR3-ANCA was significantly lower (58 versus 64 years) than that in patients with MPO-ANCA positivity $(p=0.001)$. The "sclerotic" histological class was observed in $16 \%$ of the patients with MPO-ANCA as compared to 4\% with PR3ANCA $(p<0.001)$. No other significant differences in baseline characteristics were observed, except for relatively minor differences in serum albumin and diastolic blood pressure. Median observation period with PR3-ANCA was 3.9 years (25th-75th percentiles $=0.8-7.8$ years) and 2.8 years (25th-75th percentiles $=0.4-7.3$ years) with MPO-ANCA. The total number of patient years was 1016 with PR3- and 701 with MPO-ANCA.

As demonstrated in Figure 1(a), cumulative survival without ESRD (deaths without ESRD treated as a censoring 
TABLE 1: Baseline characteristics in the total cohort and stratified for MPO- and PR3-ANCA.

\begin{tabular}{|c|c|c|c|c|}
\hline Characteristic & All, $n=358$ & MPO-ANCA, $n=165$ & PR3-ANCA, $n=193$ & $p$ value \\
\hline Males (\%) & $187(52)$ & $68(41)$ & $119(62)$ & $<0.001$ \\
\hline Mean age (SD) & $60(17)$ & $64(15)$ & $58(18)$ & 0.001 \\
\hline eGFR (SD) & $34(30)$ & $31(28)$ & $36(32)$ & 0.07 \\
\hline Serum albumin (SD) & $32(7)$ & $32(7)$ & $31(7)$ & 0.04 \\
\hline Systolic blood pressure (SD) & $142(21)$ & $144(21)$ & $141(21)$ & 0.08 \\
\hline Diastolic blood pressure (SD) & $80(11)$ & $82(11)$ & $79(11)$ & 0.05 \\
\hline Proteinuria $(\mathrm{SD})$ & $1.8(2.0)$ & $1.9(2.0)$ & $1.7(2.0)$ & 0.25 \\
\hline Percentage normal glomeruli (SD) & $36(29)$ & $33(28)$ & $38(30)$ & 0.07 \\
\hline Focal histology (\%) & $127(35)$ & $53(32)$ & $74(38)$ & 0.21 \\
\hline Mixed histology (\%) & $90(25)$ & $40(25)$ & $50(26)$ & 0.82 \\
\hline Crescentic histology (\%) & $106(30)$ & $45(27)$ & $61(32)$ & 0.32 \\
\hline Sclerotic histology (\%) & $35(10)$ & $27(16)$ & $8(4)$ & $<0.001$ \\
\hline
\end{tabular}

event) in the 0-1-year observation period was significantly better with PR3 - rather than MPO-ANCA ( $p=0.007)$. However, when the total observation period was analyzed, the difference was no longer statistically significant $(p=0.07$, Figure 1(b)). Furthermore, when cases with sclerotic histology were excluded from the analysis, survival without ESRD was basically similar in both ANCA subtypes $(p=0.34$, Figure 1(c)). One- and 5-year cumulative ESRD-free survival rates with PR3- versus MPO-ANCA stratified according to the histological classification model are shown in Table 2 and Figures 1(d)-1(g). No significant differences were observed during the analysis of the survival rates stratified for histological classification. In the Cox regression analysis, HR for ESRD with MPO- versus PR3-ANCA was 1.30 $(0.83-2.02, p=0.25)$, with adjustments for age, gender, and histological classification.

One- and 5-year cumulative ESRD-free survival (deaths without ESRD treated as an event) with PR3- versus MPO-ANCA stratified according to the histological classification model is shown in Table 3. No significant differences were observed. In the Cox regression analysis, HR for ESRD/death with MPO- versus PR3-ANCA was $1.04(0.75-1.43, p=0.83)$, with adjustments for age, sex, and histological classification.

One- and 5-year cumulative patient survival (deaths with ESRD included) with PR3- versus MPO-ANCA stratified according to the histological classification model is shown in Table 4. No significant differences were observed. In the Cox regression analysis, HR for death with $\mathrm{MPO}$ - versus PR3-ANCA was $0.77(0.53-1.13, p=0.18)$, with adjustment for age, sex, and histological classification.

\section{Discussion}

In this study, we have demonstrated that the risk of ESRD and/or death in patients with ANCA-GN is similar irrespective of PR3- or MPO-ANCA positivity when the histological picture at baseline is similar. The consequence of this finding for clinical practice and future research is that histological classification is more relevant and important than serological classification of ANCA-GN with respect to prognosis. To the best of our knowledge, no study has previously reported ANCA-serotype-specific outcomes of ANCA-GN, stratified by histological classification. Explanation for this surprising lack of data is probably that many studies involved small samples unsuitable for stratified analyses [32]. We hope our findings will encourage researchers to conduct multicenter studies or meta-analyses that will confirm our results.

Our study confirms several previous findings with respect to differences in baseline characteristics of patients with ANCA-GN according to serotype [3]. In a number of studies, the differences in male to female ratio in patients with PR3as compared to MPO-ANCA GN were identified $[3,6,7]$. A higher mean age in patients with MPO- than PR3-ANCA was also previously described [3]. Similar to our findings, sclerotic histology was significantly more frequent in patients with MPO- than PR3-ANCA in a previous study with patients recruited from Spain and the UK [33]. The probable explanation is the fewer extrarenal symptoms and findings and thus the increased delay in the diagnosis of ANCAGN in patients with MPO- than with PR3-ANCA. In some studies, patients with MPO-ANCA had higher baseline serum creatinine and a higher percentage of affected glomeruli than those with PR3-ANCA [8-10, 12-14]. We observed the same tendency in the current study. However, differences were not statistically significant. The significantly higher risk of ESRD with MPO- as compared to PR3-ANCA at 1 year of observation is also found in numerous previous studies [3, 18-20]. Here, we can document that this difference in prognosis is solely attributed to a higher fraction with sclerotic histology among patients with MPO- than PR3-ANCA.

The strength of this study is that it included a relatively large population-based study cohort with reliable registration of endpoints from high-quality national registries. However, some limitations must also be considered. We know that the treatment regimen for ANCA-GN in Norway during the study period almost exclusively 


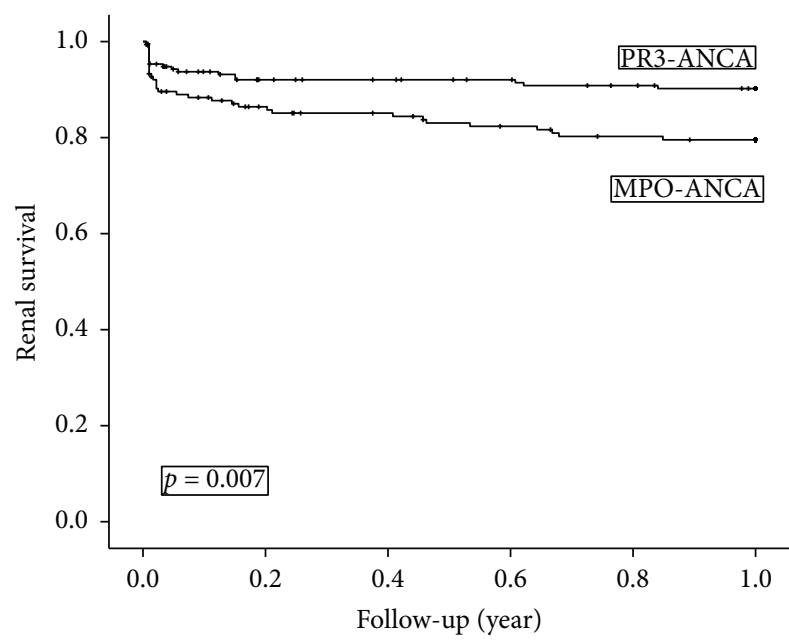

(a)

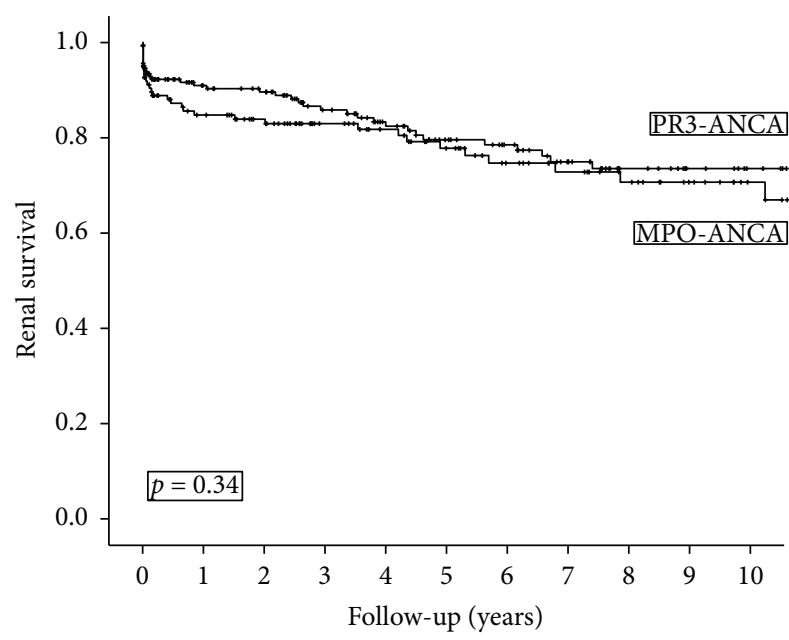

(c)

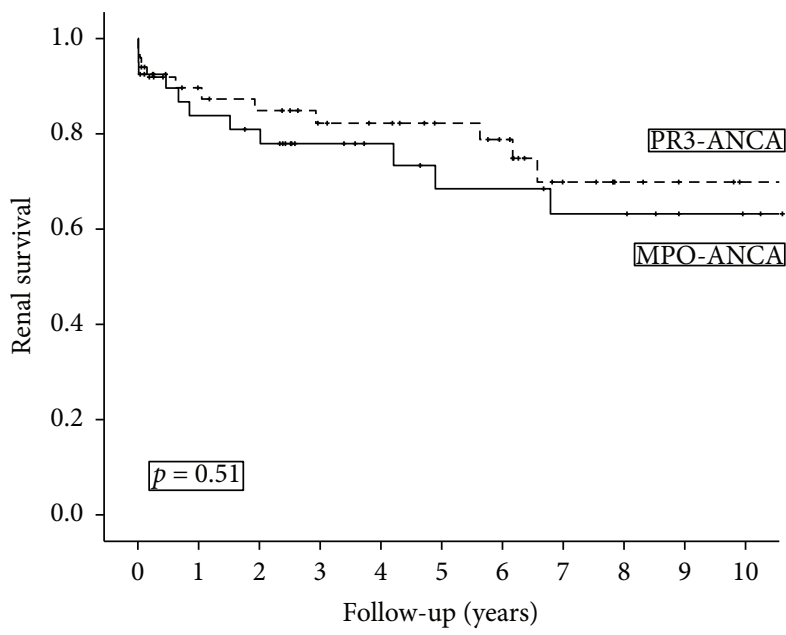

(e)

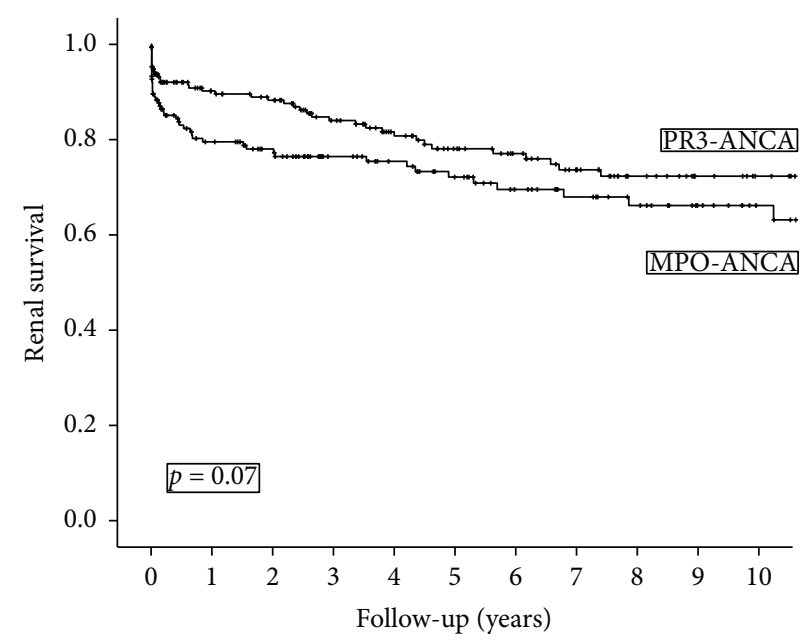

(b)

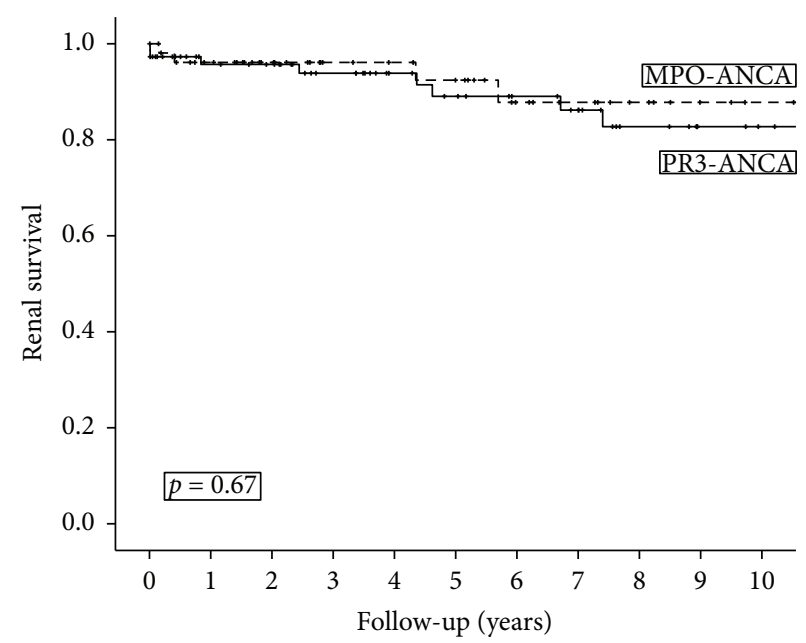

(d)

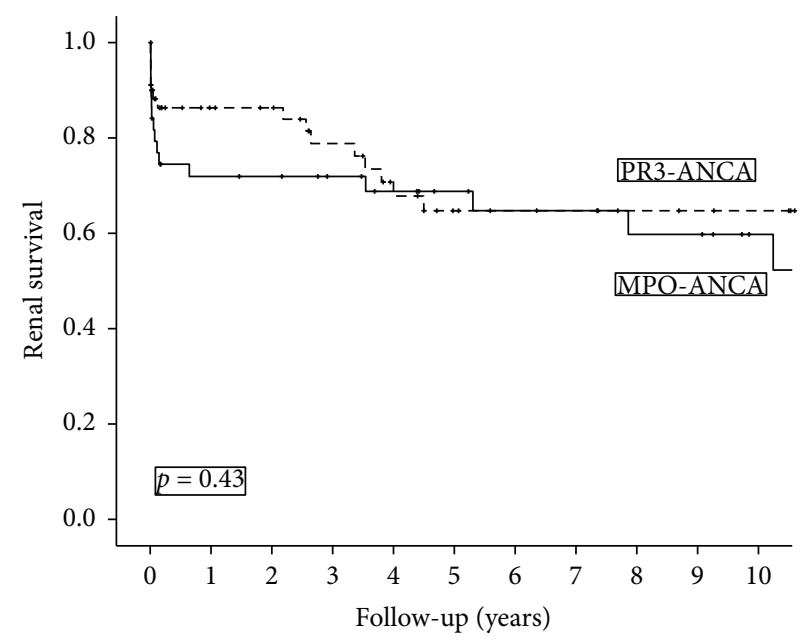

(f)

FIgURe 1: Continued. 


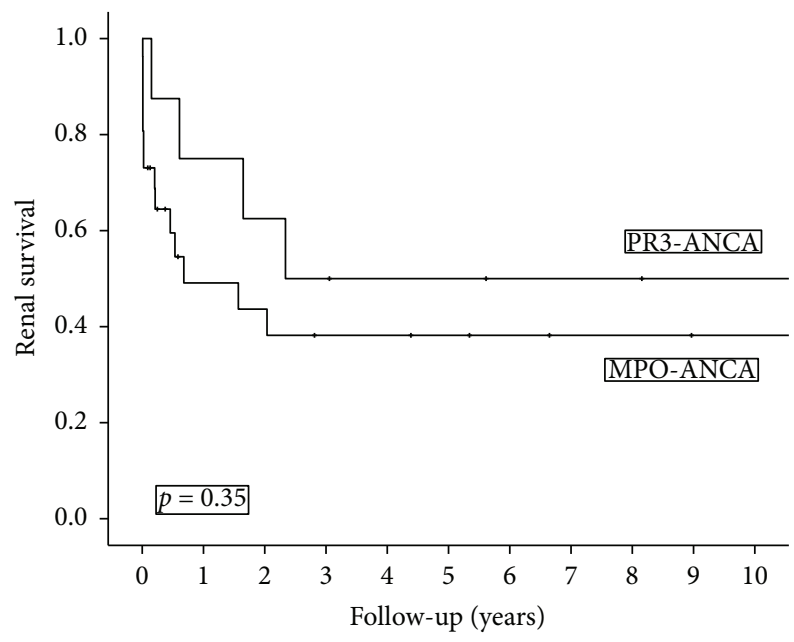

(g)

Figure 1: (a) Kaplan-Meier plot demonstrating end-stage renal disease-free survival at 0-1 after diagnosis with MPO- versus PR3-ANCA. (b) Kaplan-Meier plot demonstrating end-stage renal disease-free survival in total observation period with MPO- versus PR3-ANCA. (c) KaplanMeier plot demonstrating end-stage renal disease-free survival in total observation period with MPO- versus PR3-ANCA. Cases with sclerotic histology were excluded. (d) Kaplan-Meier plot demonstrating end-stage renal disease-free survival with MPO- versus PR3-ANCA in cases with focal histology. (e) Kaplan-Meier plot demonstrating end-stage renal disease-free survival with MPO- versus PR3-ANCA in cases with mixed histology. (f) Kaplan-Meier plot demonstrating end-stage renal disease-free survival with MPO- versus PR3-ANCA in cases with crescentic histology. (g) Kaplan-Meier plot demonstrating end-stage renal disease-free survival with MPO- versus PR3-ANCA in cases with sclerotic histology.

TABLE 2: End-stage renal disease-free survival at 1 and 5 years of follow-up with MPO- and PR3-ANCA, total cohort and stratified according to histological classification.

\begin{tabular}{|c|c|c|c|c|c|c|c|c|c|}
\hline \multirow{2}{*}{ Characteristic } & \multicolumn{2}{|c|}{$N$} & \multicolumn{2}{|c|}{ ESRD } & \multicolumn{2}{|c|}{1 year } & \multicolumn{2}{|c|}{5 years } & \multirow{2}{*}{$p$ value } \\
\hline & PR3-ANCA & MPO-ANCA & PR3-ANCA & MPO-ANCA & PR3-ANCA & MPO-ANCA & PR3-ANCA & MPO-ANCA & \\
\hline All & 193 & 165 & 41 & 46 & $90 \%$ & $80 \%$ & $78 \%$ & $72 \%$ & 0.07 \\
\hline Focal & 74 & 53 & 8 & 4 & $96 \%$ & $96 \%$ & $89 \%$ & $92 \%$ & 0.67 \\
\hline Mixed & 50 & 40 & 11 & 11 & $90 \%$ & $84 \%$ & $82 \%$ & $69 \%$ & 0.51 \\
\hline Crescentic & 61 & 45 & 18 & 17 & $86 \%$ & $72 \%$ & $65 \%$ & $69 \%$ & 0.43 \\
\hline Sclerotic & 8 & 27 & 4 & 14 & $75 \%$ & $49 \%$ & $50 \%$ & $38 \%$ & 0.35 \\
\hline
\end{tabular}

TABLE 3: Overall and end-stage renal disease-free survival at 1 and 5 years of follow-up with MPO- and PR3-ANCA, total cohort and stratified according to histological classification.

\begin{tabular}{lccccccccc}
\hline \multirow{2}{*}{ Characteristic } & \multicolumn{2}{c}{$N$} & \multicolumn{2}{c}{ ESRD/deaths } & \multicolumn{2}{c}{1 year } & \multicolumn{2}{c}{5 years } & \multicolumn{2}{c}{ r } \\
& PR3-ANCA & MPO-ANCA & PR3-ANCA & MPO-ANCA & PR3-ANCA & MPO-ANCA & PR3-ANCA & MPO-ANCA & $p$ value \\
\hline All & 193 & 165 & 84 & 81 & $76 \%$ & $69 \%$ & $62 \%$ & $55 \%$ & 0.13 \\
Focal & 74 & 53 & 25 & 16 & $84 \%$ & $87 \%$ & $72 \%$ & $71 \%$ & 0.95 \\
Mixed & 50 & 40 & 19 & 19 & $80 \%$ & $75 \%$ & $73 \%$ & $53 \%$ & 0.53 \\
Crescentic & 61 & 45 & 36 & 27 & $64 \%$ & $62 \%$ & $43 \%$ & $52 \%$ & 0.92 \\
Sclerotic & 8 & 27 & 4 & 19 & $75 \%$ & $37 \%$ & $50 \%$ & $30 \%$ & 0.16 \\
\hline
\end{tabular}

consisted of cyclophosphamide and steroids, with azathioprine substituting for cyclophosphamide for maintenance treatment after 2003 [19, 29, 34]. However, we do not know the specific treatment plans of individual patients. In a previous study using the same patient cohort, we demonstrated that outcomes were in line with those reported in other studies, indicating that treatment followed acceptable international standards [29]. Another limitation is the lack of data regarding extrarenal vasculitis manifestations. Such data are not registered in the NKBR, which focuses on renal disease.

In conclusion, we have not found ANCA-serotypespecific differences in outcomes of ANCA-GN stratified by 
TABLE 4: Patient survival at 1 and 5 years of follow-up with MPO- and PR3-ANCA, total cohort and stratified according to histological classification.

\begin{tabular}{lccccccccc}
\hline \multirow{2}{*}{ Characteristic } & \multicolumn{2}{c}{$N$} & \multicolumn{2}{c}{ Deaths } & \multicolumn{2}{c}{1 year } & \multicolumn{2}{c}{5 years } & \multicolumn{2}{c}{. } \\
& PR3-ANCA & MPO-ANCA & PR3-ANCA & MPO-ANCA & PR3-ANCA & MPO-ANCA & PR3-ANCA & MPO-ANCA & $p$ value \\
\hline All & 193 & 165 & 62 & 55 & $84 \%$ & $85 \%$ & $70 \%$ & $74 \%$ & 0.64 \\
Focal & 74 & 53 & 19 & 12 & $86 \%$ & $91 \%$ & $78 \%$ & $78 \%$ & 0.97 \\
Mixed & 50 & 40 & 17 & 12 & $86 \%$ & $82 \%$ & $72 \%$ & $74 \%$ & 0.70 \\
Crescentic & 61 & 45 & 24 & 19 & $77 \%$ & $87 \%$ & $60 \%$ & $76 \%$ & 1.00 \\
Sclerotic & 8 & 27 & 2 & 12 & $100 \%$ & $78 \%$ & $70 \%$ & $65 \%$ & 0.37 \\
\hline
\end{tabular}

histological classification. As a result, we conclude that with respect to prognosis, histological classification is more important than serologic classification in patients with ANCA-GN.

\section{Data Availability}

An anonymized version of the data file is available from the corresponding author upon request.

\section{Conflicts of Interest}

The authors declare no conflict of interest regarding the publication of this article.

\section{Acknowledgments}

The authors thank all nephrologists and pathologists in Norway who reported important data to the Norwegian Kidney Biopsy Registry. The authors thank Torbjørn Leivestad for the linkage of the data from the study cohort with that of the Norwegian Renal Registry. The authors also thank Editage (https://www.editage.com) for English language editing.

\section{References}

[1] J. C. Jennette and P. H. Nachman, "ANCA glomerulonephritis and vasculitis," Clinical Journal of the American Society of Nephrology, vol. 12, no. 10, pp. 1680-1691, 2017.

[2] C. Mukhtyar, O. Flossmann, B. Hellmich et al., "Outcomes from studies of antineutrophil cytoplasm antibody associated vasculitis: a systematic review by the European League Against Rheumatism systemic vasculitis task force," Annals of the Rheumatic Diseases, vol. 67, no. 7, pp. 1004-1010, 2008.

[3] M. Hilhorst, P. van Paassen, J. W. C. Tervaert, and for the Limburg Renal Registry, "Proteinase 3-ANCA vasculitis versus myeloperoxidase-ANCA vasculitis," Journal of the American Society of Nephrology, vol. 26, no. 10, pp. 2314-2327, 2015.

[4] P. A. Lyons, T. F. Rayner, S. Trivedi et al., "Genetically distinct subsets within ANCA-associated vasculitis," The New England Journal of Medicine, vol. 367, no. 3, pp. 214-223, 2012.

[5] G. Xie, D. Roshandel, R. Sherva et al., "Association of granulomatosis with polyangiitis (Wegener's) with HLA-DPB1* 04 and SEMA6A gene variants: evidence from genomewide analysis," Arthritis \& Rheumatism, vol. 65, no. 9, pp. 2457-2468, 2013.
[6] C. Geffriaud-Ricouard, L. H. Noël, D. Chauveau, S. Houhou, J. P. Grünfeld, and P. Lesavre, "Clinical spectrum associated with ANCA of defined antigen specificities in 98 selected patients," Clinical Nephrology, vol. 39, no. 3, pp. 125-136, 1993.

[7] M. Hilhorst, B. Wilde, P. van Breda Vriesman, P. van Paassen, J. W. Cohen Tervaert, and for the Limburg Renal Registry, "Estimating renal survival using the ANCA-associated GN classification," Journal of the American Society of Nephrology, vol. 24, no. 9, pp. 1371-1375, 2013.

[8] A. A. E. de Joode, J. S. F. Sanders, and C. A. Stegeman, "Renal survival in proteinase 3 and myeloperoxidase ANCAassociated systemic vasculitis," Clinical Journal of the American Society of Nephrology, vol. 8, no. 10, pp. 1709-1717, 2013.

[9] A. Mahr, S. Katsahian, H. Varet et al., "Revisiting the classification of clinical phenotypes of anti-neutrophil cytoplasmic antibody-associated vasculitis: a cluster analysis," Annals of the Rheumatic Diseases, vol. 72, no. 6, pp. 1003-1010, 2013.

[10] I. M. Bajema, E. C. Hagen, B. E. Hansen et al., "The renal histopathology in systemic vasculitis: an international survey study of inter- and intra-observer agreement," Nephrology Dialysis Transplantation, vol. 11, no. 10, pp. 1989-1995, 1996.

[11] R. J. Falk and J. C. Jennette, "ANCA small-vessel vasculitis," Journal of the American Society of Nephrology, vol. 8, no. 2, pp. 314-322, 1997.

[12] C. Franssen, R. Gans, C. Kallenberg, C. Hageluken, and S. Hoorntje, "Disease spectrum of patients with antineutrophil cytoplasmic autoantibodies of defined specificity: distinct differences between patients with anti-proteinase 3 and anti-myeloperoxidase autoantibodies," Journal of Internal Medicine, vol. 244, no. 3, pp. 209-216, 1998.

[13] H. A. Hauer, I. M. Bajema, H. van Houwelingen et al., "Renal histology in ANCA-associated vasculitis: differences between diagnostic and serologic subgroups," Kidney International, vol. 61, no. 1, pp. 80-89, 2002.

[14] M. Hilhorst, B. Wilde, P. van Paassen et al., "Improved outcome in anti-neutrophil cytoplasmic antibody (ANCA)associated glomerulonephritis: a 30-year follow-up study," Nephrology Dialysis Transplantation, vol. 28, no. 2, pp. 373379, 2013.

[15] S. Lionaki, E. R. Blyth, S. L. Hogan et al., "Classification of antineutrophil cytoplasmic autoantibody vasculitides: the role of antineutrophil cytoplasmic autoantibody specificity for myeloperoxidase or proteinase 3 in disease recognition and prognosis," Arthritis \& Rheumatism, vol. 64, no. 10, pp. 3452-3462, 2012.

[16] M. C. Slot, J. W. C. Tervaert, M. M. Boomsma, and C. A. Stegeman, "Positive classic antineutrophil cytoplasmic antibody (C-ANCA) titer at switch to azathioprine therapy 
associated with relapse in proteinase 3-related vasculitis," Arthritis \& Rheumatism, vol. 51, no. 2, pp. 269-273, 2004.

[17] M. Walsh, O. Flossmann, A. Berden et al., "Risk factors for relapse of antineutrophil cytoplasmic antibody-associated vasculitis," Arthritis \& Rheumatism, vol. 64, no. 2, pp. 542-548, 2012.

[18] A. J. Mohammad and M. Segelmark, "A population-based study showing better renal prognosis for proteinase 3 antineutrophil cytoplasmic antibody (ANCA)-associated nephritis versus myeloperoxidase ANCA-associated nephritis," The Journal of Rheumatology, vol. 41, no. 7, pp. 1366-1373, 2014.

[19] S. Sriskandarajah, K. Aasarød, S. Skrede, T. Knoop, A. V. Reisæter, and R. Bjørneklett, "Improved prognosis in Norwegian patients with glomerulonephritis associated with antineutrophil cytoplasmic antibodies," Nephrology Dialysis Transplantation, vol. 30, Supplement 1, pp. i67-i75, 2015.

[20] A. Tanna, L. Guarino, F. W. K. Tam et al., "Long-term outcome of anti-neutrophil cytoplasm antibody-associated glomerulonephritis: evaluation of the international histological classification and other prognostic factors," Nephrology Dialysis Transplantation, vol. 30, no. 7, pp. 1185-1192, 2015.

[21] R. A. F. de Lind van Wijngaarden, H. A. Hauer, R. Wolterbeek et al., "Clinical and histologic determinants of renal outcome in ANCA-associated vasculitis: a prospective analysis of 100 patients with severe renal involvement," Journal of the American Society of Nephrology, vol. 17, no. 8, pp. 2264-2274, 2006.

[22] C. F. M. Franssen, R. O. B. Gans, B. Arends et al., "Differences between anti-myeloperoxidase- and anti-proteinase 3associated renal disease," Kidney International, vol. 47, no. 1, pp. 193-199, 1995.

[23] H. A. Hauer, I. M. Bajema, E. C. Hagen et al., "Long-term renal injury in ANCA-associated vasculitis: an analysis of 31 patients with follow-up biopsies," Nephrology Dialysis Transplantation, vol. 17, no. 4, pp. 587-596, 2002.

[24] Z. Rihova, E. Jancova, M. Merta et al., "Long-term outcome of patients with antineutrophil cytoplasmic autoantibodyassociated vasculitis with renal involvement," Kidney \& Blood Pressure Research, vol. 28, no. 3, pp. 144-152, 2005.

[25] M. Chen, M. R. Daha, and C. G. M. Kallenberg, "The complement system in systemic autoimmune disease," Journal of Autoimmunity, vol. 34, no. 3, pp. J276-J286, 2010.

[26] A. Schreiber, H. Xiao, J. C. Jennette, W. Schneider, F. C. Luft, and R. Kettritz, "C5a receptor mediates neutrophil activation and ANCA-induced glomerulonephritis," Journal of the American Society of Nephrology, vol. 20, no. 2, pp. 289-298, 2009.

[27] W. Vogt, "Complement activation by myeloperoxidase products released from stimulated human polymorphonuclear leukocytes," Immunobiology, vol. 195, no. 3, pp. 334-346, 1996.

[28] A. E. Berden, F. Ferrario, E. C. Hagen et al., "Histopathologic classification of ANCA-associated glomerulonephritis," Journal of the American Society of Nephrology, vol. 21, no. 10, pp. 1628-1636, 2010.

[29] R. Bjorneklett, S. Sriskandarajah, and L. Bostad, "Prognostic value of histologic classification of ANCA-associated glomerulonephritis," Clinical Journal of the American Society of Nephrology, vol. 11, no. 12, pp. 2159-2167, 2016.

[30] D. Y. Chang, L. H. Wu, G. Liu, M. Chen, C. G. M. Kallenberg, and M. H. Zhao, "Re-evaluation of the histopathologic classification of ANCA-associated glomerulonephritis: a study of 121 patients in a single center," Nephrology Dialysis Transplantation, vol. 27, no. 6, pp. 2343-2349, 2012.
[31] A. Tanna and C. D. Pusey, "The histopathological classification of ANCA-associated glomerulonephritis comes of age," The Journal of Rheumatology, vol. 44, no. 3, pp. 265-267, 2017.

[32] Y. X. Chen, J. Xu, X. X. Pan et al., "Histopathological classification and renal outcome in patients with antineutrophil cytoplasmic antibodies-associated renal vasculitis: a study of 186 patients and metaanalysis," The Journal of Rheumatology, vol. 44, no. 3, pp. 304-313, 2017.

[33] L. F. Quintana, N. S. Perez, E. de Sousa et al., "ANCA serotype and histopathological classification for the prediction of renal outcome in ANCA-associated glomerulonephritis," Nephrology Dialysis Transplantation, vol. 29, no. 9, pp. 1764-9, 2014.

[34] K. Aasarød, L. Bostad, J. Hammerstrøm, S. Jørstad, and B. M. Iversen, "Renal histopathology and clinical course in 94 patients with Wegener's granulomatosis," Nephrology Dialysis Transplantation, vol. 16, no. 5, pp. 953-960, 2001. 


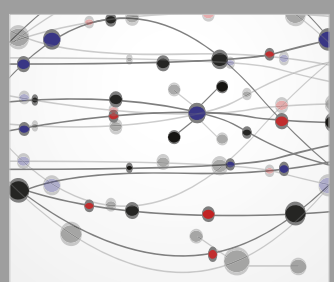

The Scientific World Journal
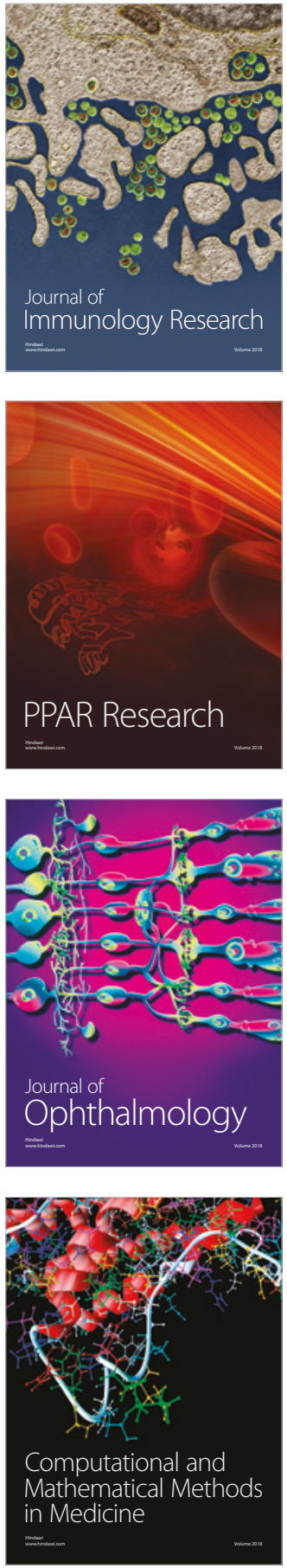

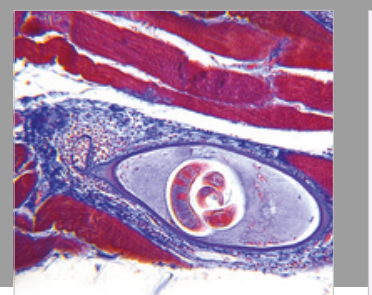

Gastroenterology Research and Practice

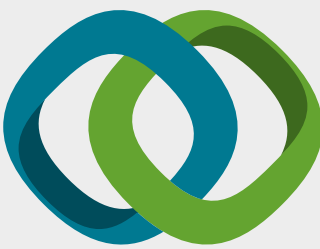

\section{Hindawi}

Submit your manuscripts at

www.hindawi.com
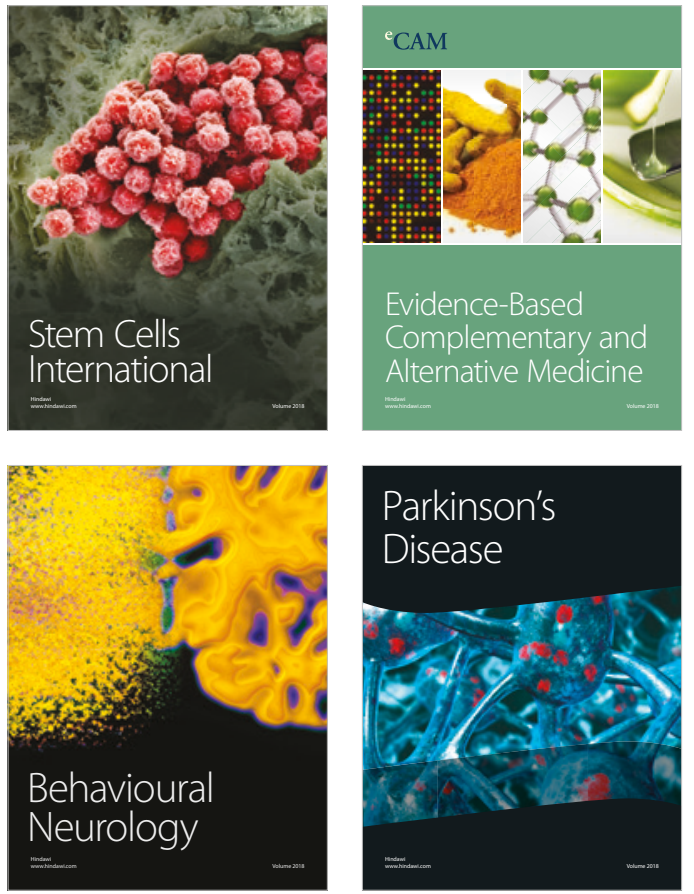

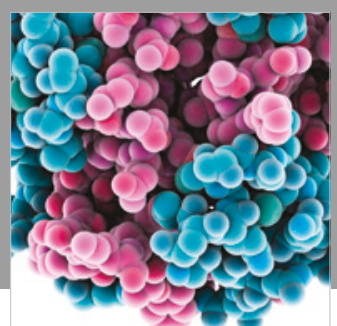

ournal of

Diabetes Research

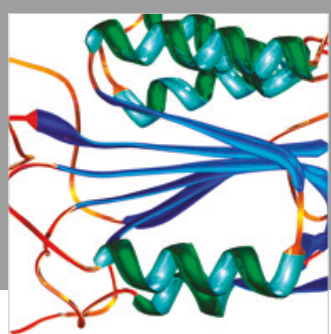

Disease Markers
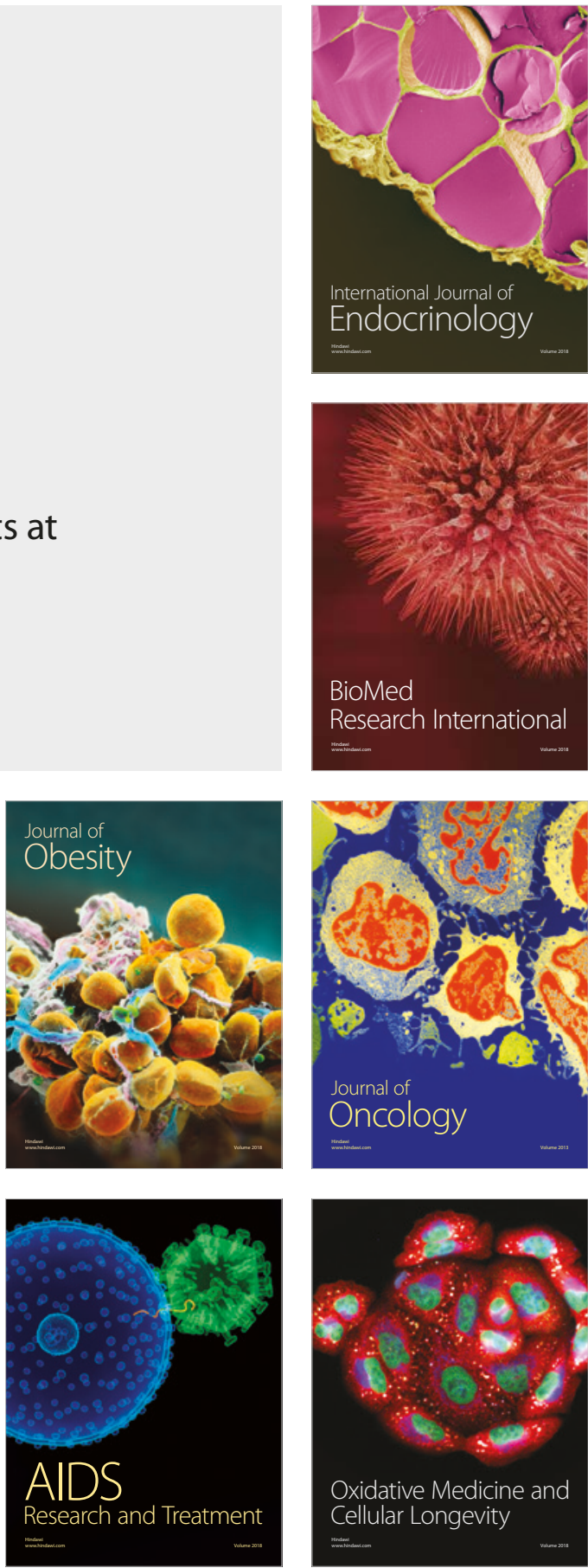